\title{
Infections contractées en voyage au Canada : réseau CanTravNet 2011-2012
}

\author{
Boggild $A K^{1,2^{*}}$, Geduld $\mathrm{J}^{3}$, Libman $\mathrm{M}^{4}$, Ward $\mathrm{BJ}^{4}$, McCarthy $\mathrm{A}^{5}$, Hajek $\mathrm{J}^{6}$, Ghesquiere $\mathbf{W}^{7}$, Vincelette $\mathrm{J}^{8}$, \\ Kuhn $\mathrm{S}^{9}$, Freedman $\mathrm{DO}^{10}$ et Kain $\mathrm{KC}^{1,11}$
}

\footnotetext{
${ }^{1}$ Unité des maladies tropicales, Département de médecine, Réseau universitaire de santé et Université de Toronto, Toronto (Ontario)

${ }^{2}$ Laboratoires de Santé publique Ontario, Santé publique Ontario, Toronto (Ontario)

${ }^{3}$ Division santé-voyage et santé liée à la migration, Agence de la santé publique du Canada, Ottawa (Ontario)

${ }^{4}$ Division des maladies infectieuses, Centre universitaire de santé McGill, Montréal (Québec)

${ }^{5}$ Clinique médicale des voyageurs internationaux, L'Hôpital d'Ottawa et Université d'Ottawa, Ottawa (Ontario)

${ }^{6}$ Division des maladies infectieuses, Hôpital général de Vancouver et Université de la Colombie-Britannique, Vancouver (Colombie-Britannique)

${ }^{7}$ Maladies infectieuses, Vancouver Island Health Authority et Université de la Colombie-Britannique, Victoria (ColombieBritannique)

${ }^{8}$ Hôpital Saint-Luc du CHUM et Université de Montréal, Montréal (Québec)

${ }^{9}$ Division des maladies infectieuses pédiatriques, Alberta Children's Hospital et Université de Calgary, Calgary (Alberta)

${ }^{10}$ Gorgas Center for Geographic Medicine, Université de l'Alabama à Birmingham, Birmingham (Alabama)

${ }^{11}$ Laboratoires SAR, Sandra Rotman Centre for Global Health, Toronto (Ontario)
}

Auteure-ressource : andrea.boggild@utoronto.ca

\section{Résumé}

Contexte : Il reste encore d'importantes lacunes à combler en ce qui concerne nos connaissances sur les maladies infectieuses que les gens contractent en voyage et l'incidence des agents pathogènes importés par les voyageurs canadiens.

Objectif : Fournir des données de surveillance actualisées sur les maladies dont souffre une cohorte de voyageurs canadiens de retour au pays et de nouveaux immigrants.

Méthodes : Les données sur les voyageurs canadiens de retour au pays et les nouveaux immigrants qui se sont présentés dans un établissement du réseau CanTravNet entre septembre 2011 et septembre 2012 ont été extraites et analysées en fonction de la destination, des symptômes, des maladies infectieuses courantes et émergentes et de la gravité de la maladie.

Résultats : Au cours de la période de l'étude, 2283 voyageurs et immigrants se sont présentés dans un établissement du réseau CanTravNet; pour $88 \%(\mathrm{~N}=2004)$ d'entre eux, on a diagnostiqué une maladie liée au voyage. Les trois principales destinations des voyageurs non-immigrants étaient l'Inde $(N=132)$, le Mexique $(N=103)$ et Cuba $(N=89)$. Cinquante et un cas de paludisme ont été importés par des voyageurs qui sont revenus malades au pays pendant la période étudiée, parmi lesquels $60 \%(\mathrm{~N}=30)$ étaient des infections à Plasmodium falciparum. Les personnes qui voyageaient pour rendre visite à des amis et à des parents représentaient $83 \%$ des cas de fièvre entérique (15/18) et $41 \%$ des cas de paludisme (21/51). Parmi les personnes ayant dû être hospitalisées, celles atteintes de paludisme étaient surreprésentées par rapport à celles qui ne l'étaient pas ( $25 \%$ contre $2,8 \% ; p<0,0001)$, de même que celles qui voyageaient pour rendre visite à des amis et à des parents par rapport à celles qui voyageaient pour d'autres raisons $(12,1 \%$ contre $2,4 \%$; 
$p<0,0001)$. Neuf nouveaux cas de VIH ont été diagnostiqués dans la cohorte, ainsi qu'un cas d'hépatite $B$ aiguë. Parmi les infections émergentes détectées chez les voyageurs, citons l'hépatite $E(N=6)$, la fièvre Chikungunya $(N=4)$ et la leishmaniose cutanée $(N=16)$. Les motifs de consultation les plus communs étaient notamment les troubles gastro-intestinaux ( $N=804)$, les problèmes dermatologiques $(N=440)$ et la fièvre $(N=287)$. Les causes spécifiques courantes de fièvre parmi les personnes étudiées ayant consulté pour cette raison étaient le paludisme ( $N=47 / 51$ cas au total), la dengue (14/18 cas au total), la fièvre entérique (14/17 cas au total), la grippe et les syndromes pseudogrippaux (15/21 cas au total). Les morsures/piqûres d'animaux étaient le dixième diagnostic le plus fréquent chez les touristes.

Interprétation : Notre analyse des données de surveillance sur les voyageurs canadiens revenus malades au pays offre une version réactualisée du spectre des maladies importées par les voyageurs canadiens. Le paludisme, la fièvre entérique, le $\mathrm{VIH}$, I'hépatite $\mathrm{B}$, l'hépatite $\mathrm{A}$, la grippe et les morsures/piqûres d'animaux font partie des maladies et des blessures contractées en voyage observées chez la cohorte qui sont évitables. Des stratégies visant à améliorer la fréquence des interventions préventives comme la chimioprophylaxie antipaludéenne, les immunisations et l'évitement des arthropodes/animaux pourraient s'avérer nécessaires.

\section{Introduction}

En 2012, les Canadiens ont dépensé 36,5 milliards de dollars dans le secteur du tourisme international, ce qui représente une hausse par rapport aux 35,9 milliards de dollars dépensés l'année précédente (1). Parmi les principales destinations touristiques des Canadiens, il y a toujours des pays tropicaux et en développement comme le Mexique, Cuba, la République dominicaine et la Jamaïque (2). En 2012, sur les 11363100 Canadiens qui ont séjourné au moins une nuit dans une destination autre que les États-Unis, près d'un tiers s'est rendu dans l'un de ces quatre pays.

Les destinations tropicales internationales font courir le risque aux voyageurs de contracter des maladies infectieuses entériques et à transmission vectorielle (3-5), dont un grand nombre peut être évité avec des interventions précises telles que la chimioprophylaxie, l'immunisation, les insectifuges, les mesures de protection personnelle et l'évitement (4). Une analyse à grande échelle des maladies diagnostiquées chez les voyageurs canadiens de retour au pays et les nouveaux immigrants pendant deux ans, qui a récemment été publiée par des membres du réseau CanTravNet (6), fournit une feuille de route épidémiologique des infections contractées en voyage aux professionnels de la santé canadiens. Ces données peuvent servir à éclairer la prise de décisions avant ou après un voyage.

Le présent rapport de surveillance consiste en une actualisation des données couvrant une période de deux ans publiées précédemment par le réseau CanTravNet (6). II met en évidence la diversité des maladies auxquelles ont été exposés les Canadiens ayant voyagé dans plus de 130 pays qui se sont rendus dans un établissement du réseau CanTravNet au cours de la période d'un an étudiée.

\section{Méthodes}

\section{Sources des données}

Six établissements canadiens de quatre provinces (Colombie-Britannique, Alberta, Ontario et Québec) faisant également partie du réseau mondial de surveillance GeoSentinel se sont regroupés pour constituer les principaux établissements du réseau CanTravNet (6). Les six établissements du Canada sont de grands centres de consultation externe par référence qui offrent principalement leurs services à la région métropolitaine de 
Vancouver/Victoria, ainsi qu'aux régions de Calgary, de Toronto, d'Ottawa et de Montréal, lesquelles représentent $47 \%$ de la population canadienne (ou une zone desservant environ 15,5 millions de personnes). Le personnel de ces établissements se compose de spécialistes de la médecine de voyage et de la médecine tropicale. II arrive souvent que les services d'urgence affiliés aiguillent immédiatement les patients vers ces centres.

Les données ont été recueillies à l'aide de la plateforme de données du réseau GeoSentinel. Ce réseau englobe 56 cliniques spécialisées en médecine tropicale/de voyage réparties sur six continents qui fournissent des données de surveillance des voyages anonymisées obtenues grâce aux cliniciens et à un questionnaire. Ces données portent sur l'ensemble des voyageurs malades examinés et sont envoyées à une base de données

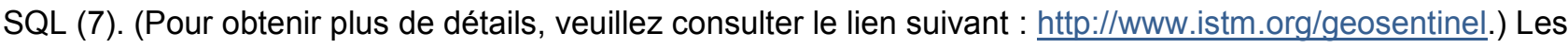
données recueillies comprennent les caractéristiques démographiques des patients, des précisions sur leur voyage récent, un historique de leurs voyages des cinq dernières années et le but de leur voyage, et indiquent s'ils ont consulté un professionnel de la santé avant leur départ. Les diagnostics définitifs sont établis par les médecins traitants, qui leur attribuent un code de diagnostic sélectionné dans une liste normalisée de plus de 500 diagnostics, dont certains portent sur l'étiologie (p. ex. Giardia) et les syndromes (p. ex. la toux). Ils entrent un code de syndrome lorsqu'ils ne peuvent pas attribuer de code étiologique pour l'une des raisons suivantes : utilisation d'un traitement empirique, maladie résolutive ou incapacité à justifier un bilan plus complet dans le cadre d'une pratique clinique de routine. Tous les établissements du réseau CanTravNet transmettent des données microbiologiquement confirmées, le cas échéant, obtenues grâce aux meilleurs tests diagnostiques nationaux de référence (diagnostics moléculaires compris) disponibles à ce moment-là. Les diagnostics « probables » se limitent aux patients présentant des signes physiques pathognomoniques (p. ex. escarre de la tique), à la réponse clinique à un traitement très particulier, ou à une présentation classique et un historique des expositions, assortis des tests en laboratoire ayant exclu les autres étiologies possibles (6). D'autres renseignements sur le réseau CanTravNet sont disponibles à l'adresse suivante $:$ http://www.istm.org/cantravnet.

\section{Définitions et classifications}

Motif du voyage le plus récent. Il est possible de choisir entre six motifs de voyage : immigration (réfugiés compris), tourisme, affaires, missionnaire/bénévole/chercheur/travailleur humanitaire, visite à des amis et à des parents et « autres " (étudiants, personnel militaire et touristes médicaux). Un voyageur rendant visite à des amis et à des parents est défini comme un immigrant appartenant à une race ou une ethnie différente de celle de la majorité de la population de son pays de résidence actuel et qui retourne dans son pays d'origine pour rendre visite à des amis et à des parents. Cette catégorie comprend également les enfants dont les parents sont nés à l'étranger (p. ex. immigrants de deuxième génération) qui retournent dans le pays d'origine de leurs parents pour rendre visite à des amis ou à des parents. On désigne habituellement comme voyageurs rendant visite à des amis et à des parents les personnes résidant dans un pays à revenu élevé qui retournent dans leur pays d'origine, à faible revenu (8). "Les touristes médicaux » renvoient aux personnes dont le principal but du voyage est de se faire soigner et qui, dans le cadre de leur voyage, développent une complication infectieuse à la suite des soins médicaux reçus ou contractent une maladie infectieuse ou non infectieuse alors qu'ils sont à l'étranger.

Les pays de destination et d'exposition sont regroupés dans 14 catégories régionales: Amérique du Nord, Amérique centrale, Caraïbes, Amérique du Sud, Europe de l'Ouest, Europe de l'Est, Moyen-Orient, Afrique du Nord, I'Afrique subsaharienne, Asie centrale du Sud, Asie du Sud-Est, Asie du Nord-Est, Australie/NouvelleZélande et Océanie.

\section{Critères d'inclusion}

On a extrait et analysé les données démographiques, cliniques et relatives au voyage portant sur les citoyens canadiens et les nouveaux immigrants au Canada recueillies après la fin de leur voyage international ou de leur 
résidence à l'étranger dans l'un des cinq établissements du réseau CanTravNet entre septembre 2011 et septembre 2012. (L'établissement de Calgary a joint le réseau GeoSentinel en 2012 et n'a donc pas transmis de données sur des cas pendant la période étudiée.) On a uniquement inclus les patients dont le diagnostic définitif était probable ou confirmé [étiologie ou syndrome précis, tel qu'il a été susmentionné (6)].

\section{Analyse descriptive}

On a géré les données extraites dans une base de données Microsoft Access, puis on les a analysées à l'aide de techniques paramétriques et non paramétriques normalisées. On a décrit les voyageurs selon l'objectif du voyage, les caractéristiques démographiques, le diagnostic, le pays d'exposition et la région de voyage. On a décrit les principaux diagnostics d'étiologie et de syndrome pour chaque objectif de voyage. On a présenté les principaux motifs de consultation selon le diagnostic représenté et les principaux pays source. On a comparé les variables nominales (p. ex. l'objectif du voyage) à l'aide de l'analyse du chi carré corrigé de Yates, et on a cherché à détecter les différences notables au niveau des variables continues ( $p$. ex. l'âge) à l'aide du test de Student et, dans le cas des paramètres anormalement répartis, à l'aide du test de Mann-Whitney. Pour une variable particulière ( $p$. ex. le but du voyage ou le diagnostic), la population de référence était l'ensemble des autres voyageurs de la cohorte sans cette variable (p. ex. atteints de paludisme ou non atteints de paludisme). On a comparé les différences entre les groupes de variables continues à l'aide de l'analyse de variance à un critère de classification ou de l'analyse de variance à un critère de classification de Kruskal-Wallis sur les rangs. On a effectué tous les calculs statistiques à l'aide du logiciel SigmaStat 2.03 (SPSS Inc., Chicago, Illinois). Le niveau de signification a été fixé à $p<0,05$.

\section{Résultats}

\section{Patients et caractéristiques démographiques}

Pour la période de surveillance étudiée, on a établi 2377 diagnostics confirmés et 338 diagnostics probables à la cohorte de 283 voyageurs qui se sont présentés dans un établissement du réseau CanTravNet. Sur les 2283 voyageurs examinés, le diagnostic était lié au voyage pour 2004 d'entre eux (87,8\%) (ci-après appelés « voyageurs revenus au pays malades ») et n'avait aucun lien avec le voyage pour 166 d'entre eux (7,3\%). En ce qui concerne les 113 voyageurs restants (4,9\%), on ne pouvait dire avec certitude si le diagnostic était lié au voyage ou non. La cohorte des 2283 voyageurs s'est présentée dans l'un des cinq établissements du réseau CanTravNet comme suit : Montréal-McGill ( $\mathrm{N}=955,41,8 \%)$, Toronto ( $\mathrm{N}=521,22,8 \%$ ), Ottawa ( $\mathrm{N}=451$, $19,8 \%)$, Montréal - Centre hospitalier de l'Université de Montréal (CHUM) ( $N=245,10,7 \%)$ et Vancouver/Victoria $(\mathrm{N}=111,4,9 \%)$. Les principales variables démographiques de la cohorte de 2004 voyageurs dont le diagnostic est lié au voyage sont résumées dans le Tableau 1. Les principaux pays de naissance des personnes nées à l'extérieur du Canada $(N=915)$ étaient l'Inde $(N=82,9 \%)$, la Chine $(N=42,4,6 \%)$, les Philippines $(\mathrm{N}=41,4,5 \%)$, la France $(\mathrm{N}=38,4,2 \%)$ et les États-Unis $(\mathrm{N}=32,3,5 \%)$, sur un total de 129 pays représentés.

Les voyageurs non-immigrants de la cohorte (c.-à-d. tous les voyageurs de la cohorte, sauf ceux voyageant aux fins d'immigration) $(N=1511 / 2004)$ pour lesquels le pays d'exposition était connu $(N=1349)$ se sont rendus dans 133 pays différents, parmi lesquels les plus fréquents étaient l'Inde $(\mathrm{N}=132,9,8 \%)$, le Mexique $(\mathrm{N}=103$, $7,6 \%$ ), Cuba ( $N=89,6,6 \%)$, la République dominicaine $(N=71,5,3 \%)$ et la Thaïlande $(N=49,3,6 \%)$. 
Tableau 1 : Caractéristiques démographiques et cliniques des 2004 voyageurs de retour au pays ou nouveaux immigrants qui se sont présentés dans un établissement du réseau CanTravNet pour le traitement d'une maladie liée à leur voyage, 2011-2012*.

\begin{tabular}{|c|c|c|c|c|c|c|c|c|c|c|c|c|c|c|}
\hline \multirow[b]{2}{*}{ Caractéristique } & \multirow{2}{*}{\multicolumn{2}{|c|}{$\begin{array}{l}\text { Tous les } \\
\text { voyageurs } \\
n=2004\end{array}$}} & \multicolumn{12}{|c|}{ But du voyage; $n^{\text {bre }}(\%)$ de voyageurs ${ }^{\ddagger}$} \\
\hline & & & \multicolumn{2}{|c|}{$\begin{array}{c}\text { Tourisme } \\
n=883\end{array}$} & \multicolumn{2}{|c|}{$\begin{array}{c}\text { Immigration } \\
n=493\end{array}$} & \multicolumn{2}{|c|}{\begin{tabular}{|c|} 
Visite à des \\
amis et à des \\
parents \\
$n=206$
\end{tabular}} & \multicolumn{2}{|c|}{$\begin{array}{c}\text { Missionnaires/ } \\
\text { bénévoles/ } \\
\text { chercheurs/ } \\
\text { travailleurs } \\
\text { humanitaires } \\
\mathrm{n}=\mathbf{2 1 2}\end{array}$} & \multicolumn{2}{|c|}{$\begin{array}{l}\text { Affaires } \\
n=160\end{array}$} & \multicolumn{2}{|c|}{$\begin{array}{l}\text { Autres }^{\S} \\
\mathrm{n}=\mathbf{5 0}\end{array}$} \\
\hline \multicolumn{15}{|l|}{ Sexe } \\
\hline Homme & 856 & 42,7 & 327 & 37,0 & 240 & 48,7 & 101 & 49,0 & 73 & 34,4 & 97 & 60,6 & 18 & 36,0 \\
\hline Femme & 1147 & 57,2 & 556 & 63,0 & 252 & 51,1 & 105 & 51,0 & 139 & 65,6 & 63 & 39,4 & 32 & 64,0 \\
\hline Inconnu & 1 & & & & 1 & 0,2 & & & & & & & & \\
\hline $\begin{array}{l}\text { Âge (ans)/Âge médian } \\
\text { (plage) }\end{array}$ & 38 & $0-87$ & 38 & $1-81$ & 39 & $1-84$ & 40 & $0-87$ & 30 & $15-79$ & 41 & $22-78$ & 25 & $9-70$ \\
\hline \multicolumn{15}{|l|}{ Type de patient } \\
\hline Patient hospitalisé & 68 & 3,4 & 13 & 1,5 & 20 & 4,1 & 25 & 12,1 & 4 & 1,9 & 5 & 3,1 & 1 & 2,0 \\
\hline Patient externe & 1936 & 96,6 & 870 & 98,5 & 473 & & 181 & & 208 & & 155 & & 49 & \\
\hline $\begin{array}{l}\text { Durée du voyage (en } \\
\text { jours)/Durée médiane }\end{array}$ & 20 & $0-3660$ & 15 & $p-366$ & NA & NA & 36 & $1-553$ & 37 & $3-3659$ & 21,5 & $1-1339$ & 46 & $3-2526$ \\
\hline \multicolumn{15}{|l|}{$\begin{array}{l}\text { Consultation médicale en } \\
\text { prévision du voyage }\end{array}$} \\
\hline Oui & 594 & 29,6 & 299 & 33,9 & NA & NA & 33 & 16,0 & 143 & 67,5 & 83 & 51,9 & 29 & 58,0 \\
\hline Non & 656 & 32,7 & 362 & 41,0 & NA & NA & 116 & 56,3 & 24 & 11,3 & 40 & 25,0 & 14 & 28,0 \\
\hline Inconnu & 754 & 37,6 & 222 & 25,1 & NA & NA & 57 & 27,7 & 45 & 21,2 & 37 & 23,1 & 7 & 14,0 \\
\hline \multicolumn{15}{|l|}{ Diagnostics de syndrome } \\
\hline Troubles gastro-intestinaux & 986 & 49,2 & 456 & 51,6 & 200 & 40,6 & 91 & 44,2 & 114 & 53,8 & 93 & 58,1 & 32 & 64,0 \\
\hline Affection dermatologique & 434 & 21,7 & 311 & 35,2 & 15 & 3,0 & 35 & 17,0 & 37 & 16,0 & 25 & 15,6 & 11 & 22,0 \\
\hline Maladie systémique fébrile & 225 & 11,2 & 70 & 7,9 & 36 & 7,3 & 60 & 29,1 & 28 & 13,2 & 28 & 17,5 & 3 & 6,0 \\
\hline Affection respiratoire & 113 & 5,6 & 41 & 4,6 & 35 & 7,1 & 15 & 7,3 & 9 & 4,2 & 12 & 7,5 & 1 & 2,0 \\
\hline \multicolumn{15}{|l|}{$\begin{array}{l}\text { Région géographique de } \\
\text { l'exposition }\end{array}$} \\
\hline Afrique subsaharienne & 444 & 22,2 & 76 & 8,6 & 141 & 28,6 & 58 & 28,2 & 105 & 49,5 & 52 & 32,5 & 22 & 44,0 \\
\hline Caraïbes & 304 & 15,2 & 245 & 27,7 & 32 & 6,5 & 14 & 6,8 & 25 & 11,8 & 13 & 8,1 & 1 & 2,0 \\
\hline Asie centrale du Sud & 278 & 13,9 & 73 & 8,3 & 104 & 21,1 & 71 & 34,5 & 13 & 6,1 & 11 & 6,9 & 9 & 18,0 \\
\hline Amérique centrale & 214 & 10,7 & 182 & 20,6 & 4 & 0,8 & 11 & 5,3 & 15 & 7,1 & 16 & 10,0 & 7 & 14,0 \\
\hline Asie du Sud-Est & 206 & 10,3 & 112 & 12,7 & 69 & 14,0 & 10 & 4,9 & 9 & 4,2 & 11 & 6,9 & 0 & 0 \\
\hline Amérique du Sud & 123 & 6,1 & 58 & 6,6 & 14 & 2,8 & 20 & 9,7 & 14 & 6,6 & 13 & 8,1 & 6 & 12,0 \\
\hline Asie du Nord-Est & 74 & 3,7 & 16 & 1,8 & 44 & 8,9 & 7 & 3,4 & 1 & 0,5 & 8 & 5,0 & 1 & 2,0 \\
\hline Amérique du Nord & 51 & 2,5 & 42 & 4,8 & 1 & 0 & 0 & 0 & 1 & 0,5 & 6 & 3,8 & 1 & 2,0 \\
\hline Europe de l'Ouest & 34 & 1,7 & 24 & 2,7 & 7 & 1,4 & 1 & 0,5 & 1 & 0,5 & 1 & 0,6 & 0 & 0 \\
\hline Europe de l'Est & 38 & 1,9 & 3 & 0,3 & 27 & 5,5 & 6 & 2,9 & 1 & 0,5 & 0 & 0 & 1 & 2,0 \\
\hline Moyen-Orient & 36 & 1,8 & 8 & 0,9 & 21 & 4,3 & 2 & 1,0 & 0 & 0 & 5 & 3,1 & 0 & 0 \\
\hline Afrique du Nord & 41 & 2,0 & 10 & 1,1 & 19 & 3,9 & 4 & 1,9 & 4 & 1,9 & 6 & 3,8 & 0 & 0 \\
\hline Australie/Nouvelle-Zélande & 5 & 0,2 & 3 & 0,3 & 0 & 0 & 0 & 0 & 0 & 0 & 1 & 0,6 & 1 & 2,0 \\
\hline Océanie & 8 & 0,4 & 4 & 0,5 & 2 & 0,4 & 0 & 0 & 2 & 0,9 & 0 & 0 & 0 & 0 \\
\hline Inconnu & 148 & 7,4 & 99 & 11,2 & 8 & 1,6 & 2 & 1,0 & 21 & 9,9 & 17 & 10,6 & 1 & 2,0 \\
\hline \multicolumn{15}{|l|}{ Pays de naissance } \\
\hline Canada & 1089 & 54,3 & 732 & 82,9 & 0 & 0 & 42 & 20,4 & 161 & 75,9 & 117 & 73,1 & 36 & 72,0 \\
\hline Hors Canada & 915 & 45,7 & 151 & 17,1 & 493 & 100,0 & 164 & $79,6^{\dagger}$ & 51 & 24,1 & 43 & 26,9 & 14 & 28,0 \\
\hline
\end{tabular}


*La cohorte comprenait 2004 voyageurs dont le diagnostic définitif était lié au voyage, 166 voyageurs dont le diagnostic n'était pas lié au voyage et 113 voyageurs pour lesquels on ne pouvait relier avec certitude le diagnostic au voyage. Cette analyse n'inclut que les voyageurs avec un diagnostic lié au voyage.

${ }^{\ddagger}$ Sauf indication contraire.

${ }^{\S}$ Comprend les étudiants $(n=42)$, le personnel militaire $(n=6)$ et les touristes médicaux $(n=2)$.

${ }^{\dagger}$ Parmi les personnes nées à l'extérieur du Canada, les personnes ayant voyagé pour rendre visite à des amis et à des parents ont été définies comme des immigrants qui appartiennent à une race ou une ethnie différente de celle de la majorité de la population de leur pays de résidence actuel et qui sont retournés dans leur pays d'origine pour rendre visite à des amis et à des parents. Ce groupe incluait également les enfants dont les parents sont nés à l'étranger (p. ex. immigrants de deuxième génération) et qui sont retournés dans le pays d'origine de leurs parents pour rendre visite à des amis et à des parents.

\section{Diagnostics}

Au total, 2402 diagnostics liés au voyage ont été posés à 2004 voyageurs revenus malades au pays. Sur ces diagnostics, 2078 étaient confirmés et 324 étaient probables. Voici les diagnostics liés au voyage les plus fréquemment établis pour les personnes voyageant aux fins d'immigration : infection tuberculeuse latente, hépatite $B$ chronique, tuberculose progressive, hépatite $C$ chronique et strongyloidïose (Tableau 2). Voici les diagnostics liés au voyage les plus fréquemment établis pour les voyageurs non-immigrants : syndrome du côlon irritable post-infectieux, diarrhée aiguë, diarrhée chronique, infections de la peau et des tissus mous et piqûre d'arthropode (Tableau 2). Le Tableau 3 dresse la liste des principaux diagnostics liés au voyage et des pays d'exposition pour les voyageurs dont le motif de consultation est de la fièvre, des symptômes gastro-intestinaux ou une maladie cutanée. Le Tableau 4 décrit les cas de paludisme chez les 2004 voyageurs qui sont revenus malades au pays.

\section{Tableau 2 : Dix principaux diagnostics d'étiologie et de syndrome par motif du voyage chez les 2004 voyageurs revenus malades au pays et dont le diagnostic définitif est lié au voyage, 2011-2012.}

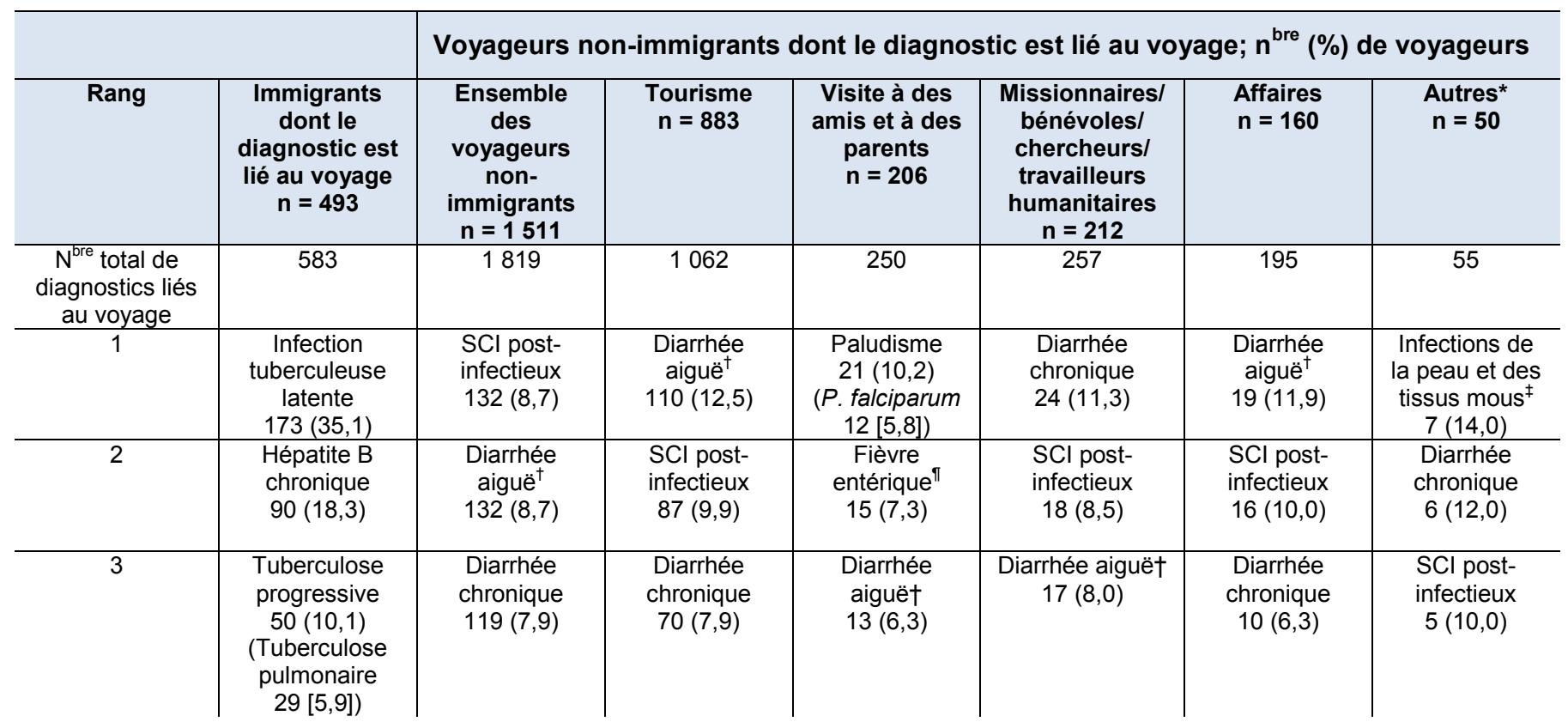




\begin{tabular}{|c|c|c|c|c|c|c|c|}
\hline 4 & $\begin{array}{l}\text { Hépatite C } \\
\text { chronique } \\
33(6,7)\end{array}$ & $\begin{array}{l}\text { Infections de } \\
\text { la peau et des } \\
\text { tissus mous }{ }^{\ddagger} \\
83(5,5)\end{array}$ & $\begin{array}{c}\text { Piqûre } \\
\text { d'arthropode } \\
65(7,4)\end{array}$ & $\begin{array}{c}\text { Diarrhée } \\
\text { chronique } \\
9(4,4)\end{array}$ & $\begin{array}{l}\text { Infections de la } \\
\text { peau et des } \\
\text { tissus mous }{ }^{\ddagger} \\
13(6,1)\end{array}$ & $\begin{array}{c}\text { Syndrome } \\
\text { viral } \\
10(6,3)\end{array}$ & $\begin{array}{l}\text { Diarrhée } \\
\text { aiguë }^{\dagger} \\
5(10,0)\end{array}$ \\
\hline 5 & $\begin{array}{c}\text { Strongyloidïose } \\
25(5,1)\end{array}$ & $\begin{array}{c}\text { Piqûre } \\
\text { d'arthropode } \\
79(5,2)\end{array}$ & $\begin{array}{l}\text { Éruption } \\
\text { cutanée } \\
52(5,9)\end{array}$ & $\begin{array}{c}\text { Maladie fébrile } \\
\text { d'une durée < } \\
3 \text { semaines } \\
7(3,4)\end{array}$ & $\begin{array}{c}\text { Paludisme } \\
12(5,7) \\
\text { (P. falciparum } \\
9[4,2])\end{array}$ & $\begin{array}{c}\text { Paludisme } \\
9(5,6) \\
\text { (P. falciparum } \\
5[3,1])\end{array}$ & $\begin{array}{c}\text { Giardiase } \\
4(8,0)\end{array}$ \\
\hline 6 & $\begin{array}{l}\text { Filariose } \\
22(4,5)\end{array}$ & $\begin{array}{l}\text { Éruption } \\
\text { cutanée } \\
74(4,9)\end{array}$ & $\begin{array}{l}\text { Infections de } \\
\text { la peau et des } \\
\text { tissus mous } \\
50(5,7)\end{array}$ & $\begin{array}{l}\text { Éruption } \\
\text { cutanée } \\
7(3,4)\end{array}$ & $\begin{array}{c}\text { Douleurs } \\
\text { abdominales/ } \\
\text { Dyspepsie } \\
12(5,7)\end{array}$ & $\begin{array}{l}\text { Éruption } \\
\text { cutanée } \\
7(4,4)\end{array}$ & $\begin{array}{c}\text { Tuberculose } \\
\text { latente } \\
3(6,0)\end{array}$ \\
\hline 7 & $\begin{array}{c}\text { Échinococcose } \\
17(3,4)\end{array}$ & $\begin{array}{c}\text { Douleurs } \\
\text { abdominales/ } \\
\text { Dyspepsie } \\
62(4,1)\end{array}$ & $\begin{array}{c}\text { Larva migrans } \\
\text { cutanée } \\
40(4,5)\end{array}$ & $\begin{array}{c}\text { SCl post- } \\
\text { infectieux } \\
6(2,9)\end{array}$ & $\begin{array}{c}\text { Tuberculose } \\
\text { latente } \\
11(5,2)\end{array}$ & $\begin{array}{c}\text { Blastocystose } \\
7(4,4)\end{array}$ & $\begin{array}{c}\text { Douleurs } \\
\text { abdominales/ } \\
\text { Dyspepsie } \\
3(6,0)\end{array}$ \\
\hline 8 & $\begin{array}{c}\text { Schistosomiase } \\
17(3,4)\end{array}$ & $\begin{array}{c}\text { Giardiase } \\
47(3,1)\end{array}$ & $\begin{array}{c}\text { Douleurs } \\
\text { abdominales/ } \\
\text { Dyspepsie } \\
39(4,4)\end{array}$ & $\begin{array}{l}\text { Infections de } \\
\text { la peau et des } \\
\text { tissus mous }{ }^{\ddagger} \\
5(2,4)\end{array}$ & $\begin{array}{c}\text { Maladie fébrile } \\
\text { d'une durée < } \\
3 \text { semaines } \\
8(3,8)\end{array}$ & $\begin{array}{c}\text { Giardiase } \\
6(3,8)\end{array}$ & $\begin{array}{c}\text { Blastocystose } \\
2(4,0)\end{array}$ \\
\hline 9 & $\begin{array}{c}\text { VIH } \\
12(2,4)\end{array}$ & $\begin{array}{c}\text { Paludisme } \\
45(3,0) \\
\text { (P. falciparum } \\
29[1,9]) \\
\end{array}$ & $\begin{array}{c}\text { Giardiase } \\
28(3,2)\end{array}$ & $\begin{array}{c}\text { Syndrome } \\
\text { viral } \\
5(2,4)\end{array}$ & $\begin{array}{c}\text { Blastocystose } \\
8(3,8)\end{array}$ & $\begin{array}{c}\text { Maladie fébrile } \\
\text { d'une durée < } \\
3 \text { semaines } \\
5(3,1)\end{array}$ & $\begin{array}{c}\text { Schistosomiase } \\
2(4,0)\end{array}$ \\
\hline 10 & $\begin{array}{c}\text { Lèpre } \\
10(2,0)\end{array}$ & $\begin{array}{c}\text { Blastocystose } \\
45(3,0)\end{array}$ & $\begin{array}{c}\text { Morsure } \\
\text { d'animal }^{\S} \\
23(2,6)\end{array}$ & $\begin{array}{c}\text { Blastocystose } \\
5(2,4)\end{array}$ & $\begin{array}{c}\text { Giardiase } \\
6(2,8)\end{array}$ & $\begin{array}{c}\text { Dientamoebiase } \\
4(2,5)\end{array}$ & $\begin{array}{c}\text { Éruption } \\
\text { cutanée } \\
2(4,0)\end{array}$ \\
\hline
\end{tabular}

P. falciparum = Plasmodium falciparum, SCI post-infectieux = Syndrome du côlon irritable post-infectieux

*Comprend les étudiants $(n=42)$, le personnel militaire $(n=6)$ et les touristes médicaux $(n=2)$.

${ }^{\ddagger}$ Comprend les diarrhées bactériennes, parasitaires et virales aiguës ainsi que les diarrhées aiguës dont l'étiologie n'est pas précisée.

${ }^{\ddagger}$ Comprend l'érysipèle, la cellulite, la furonculose, l'anthrax, les abcès cutanés, la pyodermite, l'ecthyma, l'impétigo et les mycoses cutanées superficielles.

${ }^{\S}$ Comprend les morsures de chat, de chien, de singe et d'autres animaux, de même que les griffures de singe.

"Comprend l'infection par la bactérie Salmonella enterica sérotype Typhi ou Paratyphi et les fièvres typhoïdes non précisées.

Tableau 3 : Principaux diagnostics et pays source pour des étiologies précises parmi les principaux syndromes ayant motivé une consultation chez les 2004 voyageurs revenus malades au pays dont le diagnostic définitif est lié au voyage.

\begin{tabular}{|c|c|c|c|c|}
\hline Diagnostic & \multicolumn{2}{|c|}{$N^{\text {bre }}(\%)$ de patients } & $\begin{array}{c}\mathbf{N}^{\text {bre }} \text { total de } \\
\text { la base de } \\
\text { données } \\
\text { (liés au } \\
\text { voyage) }\end{array}$ & $\begin{array}{l}\text { Trois }^{£} \text { principaux pays } \\
\text { source du diagnostic }\end{array}$ \\
\hline \multicolumn{5}{|l|}{$\begin{array}{l}\text { Motif de consultation : fièvre } \\
(n=287)\end{array}$} \\
\hline Paludisme & 47 & 92.2 & 51 & Inde, Nigeria, Sierra Leone \\
\hline Plasmodium falciparum & 28 & 93.3 & 30 & Nigeria, Haïti \\
\hline $\begin{array}{l}\text { Paludisme grave ou accès } \\
\text { pernicieux à forme cérébrale }\end{array}$ & 1 & 100.0 & 1 & \\
\hline Plasmodium vivax & 11 & 91.7 & 12 & Inde, Pakistan, Afghanistan \\
\hline Espèce de Plasmodium inconnue & 6 & 100.0 & 6 & \\
\hline Plasmodium ovale & 1 & 50.0 & 2 & Nigeria, Soudan du Sud \\
\hline
\end{tabular}




\begin{tabular}{|c|c|c|c|c|}
\hline Dengue & 14 & 77.8 & 18 & $\begin{array}{l}\text { Vietnam, Thaïlande, } \\
\text { Guyane }\end{array}$ \\
\hline Tuberculose progressive & 17 & 29.8 & 57 & $\begin{array}{l}\text { Inde, Philippines, Chine, } \\
\text { Vietnam }\end{array}$ \\
\hline Pulmonaire & 7 & 20.6 & 34 & \\
\hline Extrapulmonaire & 10 & 43.5 & 23 & \\
\hline Fièvre entérique & 14 & 82.4 & 17 & Inde, Pakistan \\
\hline $\begin{array}{l}\text { Salmonella enterica sérotype } \\
\text { Typhi }\end{array}$ & 7 & 77.8 & 9 & \\
\hline $\begin{array}{l}\text { Salmonella enterica sérotype } \\
\text { Paratyphi }\end{array}$ & 4 & 100.0 & 4 & \\
\hline Fièvre typhoïde, sans précision & 3 & 75.0 & 4 & \\
\hline $\begin{array}{l}\text { Grippe/Syndromes } \\
\text { pseudogrippaux }\end{array}$ & 15 & 71.4 & 21 & $\begin{array}{l}\text { Pérou, Inde, République } \\
\text { dominicaine }\end{array}$ \\
\hline $\begin{array}{l}\text { Infection des voies respiratoires } \\
\text { supérieures }\end{array}$ & 9 & 45.0 & 20 & $\begin{array}{l}\text { Inde, République } \\
\text { dominicaine }\end{array}$ \\
\hline Pneumonie & 9 & 56.3 & 16 & Chine, Thaïlande \\
\hline Pneumonie lobaire & 7 & 53.8 & 13 & \\
\hline Pneumonie atypique & 2 & 66.7 & 3 & \\
\hline Infection urinaire aiguë & 5 & 50.0 & 10 & Inde \\
\hline $\begin{array}{l}\text { Rickettsiose, fièvre pourprée des } \\
\text { montagnes Rocheuses }^{\dagger}\end{array}$ & 7 & 87.5 & 8 & $\begin{array}{l}\text { Afrique du Sud, Inde, } \\
\text { Malawi, Namibie }\end{array}$ \\
\hline Fièvre Chikungunya & 2 & 50.0 & 4 & $\begin{array}{l}\text { Inde, Pakistan, Cambodge, } \\
\text { Kenya }\end{array}$ \\
\hline Brucellose & 1 & 33.3 & 3 & $\begin{array}{l}\text { République dominicaine, } \\
\text { Iraq, Pérou }\end{array}$ \\
\hline \multicolumn{5}{|l|}{$\begin{array}{l}\text { Motif de consultation : troubles } \\
\text { gastro-intestinaux }(n=804)\end{array}$} \\
\hline Diarrhée aiguë ${ }^{\mp}$ & 128 & 96.2 & 133 & $\begin{array}{l}\text { Mexique, Inde, République } \\
\text { dominicaine }\end{array}$ \\
\hline $\begin{array}{l}\text { Syndrome du côlon irritable post- } \\
\text { infectieux }\end{array}$ & 133 & 100.0 & 133 & Inde, Cuba, Mexique \\
\hline Diarrhée chronique & 122 & 99.2 & 123 & Cuba, Inde, Mexique \\
\hline Giardiase & 42 & 87.5 & 48 & Inde, Cambodge, Ghana \\
\hline Dientamoebiase & 27 & 90.0 & 30 & $\begin{array}{l}\text { Mexique, République } \\
\text { dominicaine }\end{array}$ \\
\hline Campylobactériose & 16 & 100.0 & 16 & $\begin{array}{l}\text { Pérou, Indonésie, Inde, } \\
\text { Cambodge }\end{array}$ \\
\hline Cryptosporidiose, cyclosporose & 8 & 80.0 & 10 & États-Unis, Costa Rica \\
\hline $\begin{array}{l}\text { Amibiase causée par Entamoeba } \\
\text { histolytica }^{\S}\end{array}$ & 4 & 100.0 & 4 & $\begin{array}{l}\text { République dominicaine, } \\
\text { Inde, Turquie }\end{array}$ \\
\hline \multicolumn{5}{|l|}{$\begin{array}{l}\text { Motif de consultation : affection } \\
\text { dermatologique }(n=440)\end{array}$} \\
\hline $\begin{array}{l}\text { Infection de la peau et des tissus } \\
\text { mous }^{\star *}\end{array}$ & 60 & 72.3 & 83 & Cuba, Inde, États-Unis \\
\hline Piqûre d'arthropode & 77 & 97.5 & 79 & $\begin{array}{l}\text { États-Unis, République } \\
\text { dominicaine, Inde }\end{array}$ \\
\hline Insecte & 60 & 96.8 & 62 & \\
\hline Tique/araignée & 17 & 100.0 & 17 & \\
\hline Éruption cutanée & 70 & 95.9 & 73 & $\begin{array}{l}\text { Cuba, Mexique, République } \\
\text { dominicaine }\end{array}$ \\
\hline
\end{tabular}




\begin{tabular}{l|c|c|c|l} 
Dermatite atopique & 19 & 100.0 & 19 & \\
\hline Dermite de contact & 12 & 100.0 & 12 & \\
\hline Réaction aux médicaments & 2 & 66.7 & 3 & \\
\hline Réaction de photosensibilité & 7 & 100.0 & 7 & \\
\hline Éruption cutanée inconnue & 23 & 100.0 & 23 & \\
\hline Éruption urticarienne & 8 & 80.0 & 10 & \\
\hline Larva migrans cutanée & 43 & 100.0 & 43 & $\begin{array}{l}\text { Jamaïque, Thaïlande, } \\
\text { Mexique }\end{array}$ \\
\hline Morsure d'animal & & & 28 & $\begin{array}{l}\text { Thaïlande, Indonésie, } \\
\text { Mexique, Costa Rica, Chili }\end{array}$ \\
\hline Leishmaniose cutanée & 22 & 78.6 & 28 & $\begin{array}{l}\text { Costa Rica, Mexique, } \\
\text { Afghanistan }\end{array}$ \\
\hline Envenimation marine & 16 & 100.0 & 16 & États-Unis, Mexique \\
\hline
\end{tabular}

*Les pourcentages sont calculés par rapport au nombre total dans la base de données. Un voyageur revenu malade au pays peut consulter pour plusieurs motifs.

${ }^{\dagger}$ Comprend l'infection à Rickettsia africae, $R$. conorii et $R$. rickettsii.

${ }^{\ddagger}$ Comprend les diarrhées bactériennes, parasitaires et virales aiguës ainsi que les diarrhées aiguës dont la cause n'est pas précisée.

${ }^{\S}$ Comprend les amibiases intestinales et extraintestinales.

"Comprend les poux, les puces, les punaises, les moustiques, les mouches et les moucherons.

"Comprend l'érysipèle, la cellulite, la furonculose, l'anthrax, les abcès cutanés, la pyodermite, l'ecthyma, l'impétigo et les mycoses cutanées superficielles.

${ }^{\dagger+}$ Comprend les morsures de chat, de chien, de singe et d'autres animaux.

${ }^{£}$ Lorsque seuls un ou deux pays sont indiqués, cela indique une égalité entre plus de trois pays pour la deuxième ou la troisième place.

\section{Tableau 4 : Cas de paludisme chez les 2004 voyageurs revenus malades au pays dont le diagnostic} est lié au voyage, par objectif de voyage.

\begin{tabular}{|c|c|c|c|c|c|c|c|c|c|}
\hline \multicolumn{10}{|c|}{ Type de paludisme; $\mathbf{n}^{\text {bre }}$ de cas } \\
\hline Motif du voyage & $\begin{array}{c}\mathrm{N}^{\text {bre }} \text { total } \\
\text { de cas }\end{array}$ & P. falciparum & $\begin{array}{l}\text { Paludisme } \\
\text { grave ou } \\
\text { accès } \\
\text { pernicieux } \\
\text { à forme } \\
\text { cérébrale }\end{array}$ & P. vivax & P. ovale & $\begin{array}{l}\text { Espèce de } \\
\text { Plasmodium } \\
\text { inconnue }\end{array}$ & $\begin{array}{c}\text { Trois }^{\ddagger} \\
\text { principaux } \\
\text { pays } \\
\text { d'exposition }\end{array}$ & $\begin{array}{c}\text { Ont reçu } \\
\text { des } \\
\text { conseils } \\
\text { avant leur } \\
\text { voyage }\end{array}$ & $\begin{array}{l}\text { Ont reçu une } \\
\text { prophylaxie }\end{array}$ \\
\hline Tous $(n=2004)$ & 51 & 30 & 1 & 12 & 2 & 6 & $\begin{array}{c}\text { Voir le } \\
\text { Tableau } 3 \\
\end{array}$ & $\begin{array}{c}10 \\
(19,6) \\
\end{array}$ & $3^{\dagger}$ \\
\hline $\begin{array}{l}\text { Tourisme } \\
(\mathrm{n}=883)\end{array}$ & 3 & 2 & 0 & 0 & 0 & 1 & $\begin{array}{l}\text { Gabon, } \\
\text { Ghana, } \\
\text { Thaïlande } \\
\end{array}$ & $\begin{array}{c}2 \\
(66,7)\end{array}$ & 0 \\
\hline $\begin{array}{l}\text { Immigration } \\
(n=493)\end{array}$ & 6 & 2 & 0 & 4 & 0 & 0 & $\begin{array}{c}\text { Inde, } \\
\text { Afghanistan, } \\
\text { Nigeria, } \\
\text { Gabon } \\
\end{array}$ & S.O. & S.o. \\
\hline $\begin{array}{l}\text { Visite à des amis } \\
\text { et à des parents } \\
(\mathrm{n}=206)\end{array}$ & 21 & 12 & 0 & 8 & 0 & 1 & $\begin{array}{c}\text { Inde, } \\
\text { Pakistan, } \\
\text { Nigeria } \\
\end{array}$ & $\begin{array}{c}3 \\
(14,3)\end{array}$ & 1 \\
\hline $\begin{array}{l}\text { Missionnaire/ } \\
\text { bénévole/ } \\
\text { chercheur/ } \\
\text { travailleur } \\
\text { humanitaire } \\
\text { ( } \mathrm{n}=212)\end{array}$ & 12 & 9 & 1 & 0 & 1 & 1 & $\begin{array}{l}\text { Haïti, Côte } \\
\text { d'Ivoire, } \\
\text { Cameroun }\end{array}$ & $\begin{array}{c}3 \\
(25,0)\end{array}$ & 2 \\
\hline Affaires $(n=160)$ & 9 & 5 & 0 & 0 & 1 & 3 & Sierra Leone & $\begin{array}{c}2 \\
(22,2)\end{array}$ & 0 \\
\hline $\begin{array}{l}\text { Autres }^{*} \\
(\mathrm{n}=50)\end{array}$ & 0 & 0 & 0 & 0 & 0 & 0 & s.o. & S.O. & S.o. \\
\hline
\end{tabular}

S.O. $=$ Sans objet, $P$. falciparum $=$ Plasmodium falciparum .

${ }^{*}$ Comprend les étudiants $(n=42)$, le personnel militaire $(n=6)$ et les touristes médicaux $(n=2)$. 
${ }^{\dagger}$ Comprend deux voyageurs qui ont oublié de prendre des doses de doxycycline au cours de leur voyage ou qui se sont retrouvés à court de doxycycline avant la fin de leur voyage.

${ }^{\ddagger}$ Lorsque seuls un ou deux pays sont indiqués, cela signifie qu'il y a égalité entre plus de trois pays pour la deuxième ou la troisième place.

Le paludisme était le diagnostic précis le plus fréquemment posé chez les personnes ayant voyagé pour rendre visite à des amis ou à des parents, et le cinquième diagnostic le plus courant chez les voyageurs d'affaires et les missionnaires, les chercheurs, les bénévoles et les travailleurs humanitaires (Tableau 2). Le paludisme était surreprésenté chez les voyageurs ayant rendu visite à des amis et à des parents $(p<0,0001)$ et les voyageurs d'affaires $(p=0,02)$ par rapport aux autres types de voyageurs. Le paludisme était également surreprésenté chez les hommes $(p=0,0003)$. Les deux cas de paludisme qui ont été diagnostiqués chez de jeunes voyageurs revenus malades au pays ont été causés par $P$. falciparum et ont touché des enfants qui voyageaient pour rendre visite à des amis et à des parents. Parmi les voyageurs atteints de paludisme, $20 \%$ avaient consulté un professionnel de la santé avant le voyage, mais seulement trois avaient suivi une prophylaxie antipaludéenne (Tableau 4). Un quart $(\mathrm{N}=13)$ des voyageurs revenus avec le paludisme ont dû être hospitalisés, contre seulement 2,8\% $(N=55)$ de ceux n'ayant pas le paludisme $(p<0,0001)$. Si l'Afrique subsaharienne reste la principale région source des cas de paludisme importés au Canada (33/51 cas; $64,7 \%$ ), I'Inde était le principal pays d'exposition (8/51 cas, 15,7\%). Parmi les voyageurs d'affaires atteints de paludisme, 8 sur $9(88,9 \%)$ ont contracté la maladie en Afrique occidentale ou au Soudan du Sud.

Outre le paludisme, la fièvre entérique était également surreprésentée chez les personnes ayant voyagé pour rendre visite à des amis et à des parents $(p<0,0001)$ par rapport aux autres types de voyageurs. Les cas de fièvre entérique due à la bactérie Salmonella enterica sérotype Typhi $(N=2)$ et Paratyphi $(N=2)$, ainsi que de virus de l'hépatite $E(N=1)$ et de virus de l'hépatite $A(N=1)$ étaient tous représentés chez les enfants qui avaient rendu visite à des amis et à des parents. La proportion de voyageurs revenus malades au pays après avoir rendu visite à des amis et à des parents qui ont dû être hospitalisés en raison de la maladie contractée en voyage $(12,1 \%)$ était plus de cinq fois supérieure à celle des voyageurs revenus malades au pays qui n'avaient pas rendu visite à des amis et à des parents $(2,4 \%)(p<0,0001)$. Les voyageurs qui avaient rendu visite à des amis et à des parents étaient également ceux qui, en proportion, avaient le moins consulté un professionnel de la santé avant leur voyage parmi l'ensemble des voyageurs non-immigrants revenus malades au pays $(p<0,0001)$ (Tableau 1). De plus, la durée du voyage des personnes qui avaient rendu visite à des amis et à des parents était plus longue que celle des personnes qui ne l'avaient pas fait (durée médiane de 36 jours contre 18; $p<0,001$ ).

D'autres maladies émergentes, potentiellement mortelles et à déclaration obligatoire étaient représentées au sein de la cohorte de voyageurs revenus malades au pays. Il y avait neuf cas d'infection aiguë (fébrile) au VIH ou d'infection au $\mathrm{VIH}$ récemment diagnostiquée, parmi lesquels trois concernaient des voyageurs non immigrants, ainsi qu'un cas unique d'hépatite $B$ aiguë chez un touriste. L'hépatite $E$ est une infection émergente chez les voyageurs. On a diagnostiqué six cas dans cette cohorte de patients, dont $4(67 \%)$ concernaient des hommes; trois $(50 \%)$ étaient des voyageurs ayant rendu visite à des amis et à des parents, et deux $(33 \%)$ ont nécessité une hospitalisation. Les personnes atteintes d'hépatite $E$ avaient entre 4 et 63 ans. Dans cinq des six cas de virus de l'hépatite E, la maladie a été contractée en Asie centrale du Sud ou en Asie du Sud-Est. Trois cas de brucellose ont été diagnostiqués chez des immigrants et des voyageurs ayant rendu visite à des amis et à des parents. La durée du séjour était connue pour l'un des cas : elle était de 177 jours, ce qui concorde avec la longue durée d'exposition habituellement associée à la brucellose contractée pendant un voyage.

La larva migrans cutanée semble être une maladie émergente chez les voyageurs revenus malades au pays, avec 27 des 43 cas (62,8 \%) observés chez des voyageurs de retour des Caraïbes. La Jamaïque était le premier pays d'exposition pour la larva migrans cutanée, avec 18 personnes $(42 \%)$ ayant contracté cette affection sur l'île. Quatre-vingt-treize pour cent des cas de larva migrans cutanée $(N=40)$ concernaient des touristes ayant effectué de courts voyages (durée médiane du séjour : 8,5 jours). Sur les 16 cas de leishmaniose cutanée, $69 \%$ 
étaient importés d'Amérique du Sud ou d'Amérique centrale et, plus précisément, $50 \%$ des cas avaient contracté la maladie au Costa Rica.

\section{Discussion}

L'analyse des données de surveillance sur les voyageurs revenus malades au pays qui se sont présentés dans un établissement du réseau CanTravNet entre septembre 2011 et septembre 2012 a mis en lumière le spectre des maladies contractées en voyage qui ont été observées dans ces établissements. Ces données constituent une actualisation du plus grand rapport de surveillance préparé sur les maladies contractées par les voyageurs canadiens (2009-2011) (6).

\section{Des maladies potentiellement mortelles peuvent être observées chez les voyageurs}

\section{revenus malades au pays}

Le paludisme demeure la première cause précise de fièvre chez les voyageurs revenus malades au pays $(9,10)$ et dans cette étude, la maladie a été causée par le parasite $P$. falciparum, potentiellement mortel, dans $60 \%$ des cas. Les voyageurs revenus malades au pays qui sont atteints de paludisme sont huit fois plus susceptibles d'être hospitalisés que ceux ayant reçu un autre diagnostic. Le paludisme est une infection évitable. Pourtant, parmi les patients atteints de paludisme qui ont voyagé dans une région à risque connue, seuls $20 \%$ avaient pris conseil avant leur voyage et seuls $6 \%$ avaient suivi une chimioprophylaxie antipaludéenne.

II a déjà été démontré que les voyageurs sont plus susceptibles d'être exposés au sang et aux liquides organiques en voyage que chez eux $(11,12)$. Nous avons relevé neuf cas d'infection aiguë au VIH ou d'infection au $\mathrm{VIH}$ récemment diagnostiquée, dont un tiers concernait des voyageurs non immigrants, ainsi qu'un cas d'hépatite $B$ aiguë chez un touriste. L'hépatite $B$ en particulier demeure un risque pour les voyageurs internationaux, bien que la maladie soit presque entièrement évitable par la vaccination (13-15). Nos données rappellent l'importance des stratégies avant le voyage qui visent à réduire la probabilité d'exposition au sang et aux liquides organiques pendant le voyage (16), outre les stratégies qui atténuent le risque de maladies à transmission vectorielle d'origine alimentaire ou hydrique.

Les morsures d'animaux étaient le dixième diagnostic le plus fréquent chez les touristes, qui en ont été victimes dans des pays comme la Thaïlande, l'Indonésie, le Mexique, le Costa Rica et le Chili, lesquels n'ont pas toujours à disposition de l'immunoglobuline antirabique humaine ou un vaccin qui réponde aux normes minimales de puissance fixées par l'Organisation mondiale de la Santé (OMS) (17). Comme la rage est presque toujours mortelle, il est essentiel de garantir un accès rapide à une prophylaxie post-exposition complète, mais celle-ci est rarement disponible dans les régions rurales des pays en développement (17). La prévention des morsures d'animaux élimine le risque de rage et le besoin d'un accès à une prophylaxie post-exposition. Elle devrait donc, avec l'immunisation antirabique, être une intervention que l'on cherche à effectuer en prévision d'un voyage lors d'une consultation.

\section{Les voyageurs qui rendent visite à des amis et à des parents courent un risque}

On sait que les voyageurs qui rendent visite à des amis ou à des parents contractent plus fréquemment des maladies liées au voyage que les autres, probablement parce qu'ils ont tendance à séjourner dans des habitations locales et à partir pour de plus longues durées, et qu'ils peuvent ne pas avoir conscience des risques sanitaires que présente un voyage dans leur pays d'origine $(3,4,7,8,18)$. Même si cette cohorte ne comptait que $10 \%$ de voyageurs ayant rendu visite à des amis et à des parents, ceux-ci représentaient $83 \%$ des cas de fièvre entérique et $41 \%$ de cas de paludisme. De plus, la proportion de voyageurs ayant rendu à des amis et à des parents qui ont dû être hospitalisés était cinq fois supérieure à celle des voyageurs revenus malades au pays qui 
n'avaient pas rendu visite à des amis et à des parents, ce qui peut simplement signifier que les personnes qui rendent visite à des amis et à des parents cherchent davantage à se faire soigner en cas de maladie grave que d'étiologie bénigne, et non qu'ils sont plus susceptibles de contracter des maladies graves. Les personnes qui ont rendu visite à des amis et à des parents ont voyagé plus longtemps en moyenne, mais ce sont elles qui, parmi tous les voyageurs, ont le moins cherché à obtenir des conseils avant leur voyage. Les données soulignent les caractéristiques uniques de ces voyageurs, de même que la nécessité d'élaborer au plus vite des stratégies en vue de minimiser leur morbidité liée au voyage.

\section{Il est difficile de prévenir les infections émergentes chez les voyageurs}

L'hépatite $E$ est un virus d'origine hydrique qui cause d'importantes épidémies en Amérique centrale, en Afrique subsaharienne, au Moyen-Orient et en Asie (19, 20). Dans notre cohorte, $83 \%$ des personnes atteintes ont contracté la maladie dans le sous-continent indien ou en Asie du Sud-Est, et une fois encore, les personnes ayant rendu visite à des amis et à des parents étaient le type de voyageurs le plus représenté, ce qui laisse à penser que, comme c'est le cas pour l'hépatite $A$ et la fièvre entérique (21), un séjour plus long, dans un milieu rural et chez l'habitant, peut être un facteur de risque pour l'hépatite $E$. L'infection par le virus de l'hépatite $E$ est particulièrement dangereuse pour les femmes enceintes (20), surtout au troisième trimestre, où la mortalité maternelle associée atteint $25 \%$ (22). Les voyageuses enceintes devraient donc être informées de ce risque lorsqu'elles se rendent dans des pays où la maladie est endémique (19), et il faudrait insister auprès d'elles sur les précautions à prendre par rapport à l'eau et aux aliments.

La fièvre Chikungunya est récemment apparue dans les Amériques (23), et on prévoit que les médecins canadiens observeront de plus en plus souvent cette maladie (24). Les mesures de prévention du virus Chikungunya consistent à éviter les moustiques, principalement en utilisant des insectifuges, qui rebutent souvent les voyageurs (25-28). La larva migrans cutanée, bien qu'elle ne soit pas potentiellement mortelle, entraîne une très forte morbidité, une perte de productivité et donne lieu à des rendez-vous médicaux coûteux en raison de la gravité de prurit et de l'absence d'accès immédiat aux seuls médicaments efficaces, l'albendazole et l'ivermectine, qui ne sont pas homologués au Canada à l'heure actuelle. Le Canada ne peut acquérir ces médicaments que par l'intermédiaire du Programme d'accès spécial de Santé Canada, qui nécessite de remplir un formulaire papier ou électronique de demande d'approbation. Le délai de traitement est d'au moins une semaine pour chaque demande (http://www.hc-sc.gc.ca/dhp-mps/acces/drugs-drogues/sapg3 pasg3-fra.php). Les mesures de prévention de la larva migrans cutanée consistent à éviter d'exposer ses pieds ou sa peau nue au sable, ce qui est difficile à respecter dans les lieux balnéaires où le micro-organisme responsable de l'infection est prévalent. La leishmaniose cutanée, une maladie à transmission vectorielle émergente chez les voyageurs (29), est difficile à prévenir, car elle requiert d'éviter toute piqûre à des endroits où la température et le taux d'humidité ambiants sont élevés, et où l'utilisation de vêtements couvrants et d'insectifuges peut être considérée comme gênante (2528). Les voyageurs peuvent se protéger dans une certaine mesure en utilisant des moustiquaires traitées à l'insecticide et en dormant à plusieurs pieds au-dessus du sol.

\section{Limites}

L'analyse des données du réseau CanTravNet a plusieurs limites. Ce rapport porte uniquement sur les voyageurs revenus malades au pays qui se sont présentés dans un centre du réseau CanTravNet; les conclusions pourraient donc manquer de validité externe. II est à noter qu'un grand nombre de maladies détectées chez les personnes qui voyagent aux fins d'immigration, y compris la tuberculose latente, l'hépatite B et l'hépatite $C$, pourraient avoir été diagnostiquées grâce au dépistage des personnes à risque provenant de zones endémiques; elles ne peuvent donc pas être liées de façon certaine au voyage. Les voyageurs atteints de maladies ayant une période d'incubation très courte ou très longue peuvent avoir cherché à se faire soigner à différents endroits : il 
est donc difficile de relier de façon certaine ces diagnostics avec le voyage. De la même façon, les voyageurs malades qui reviennent d'endroits considérés comme présentant de faibles risques pourraient être sousreprésentés dans la base de données du réseau CanTravNet. Les données ne permettent pas d'estimer le taux d'incidence d'une maladie précise ou de chiffrer précisément les risques de contracter ladite maladie à une destination $(7,30)$. Les différences entre les protocoles de dépistage en place pour les nouveaux immigrants et les réfugiés dans les établissements peuvent avoir conduit certains d'entre eux à diagnostiquer bien plus ou bien moins de cas que d'autres centres pour certaines pathologies. Cinquante-trois pour cent des cas ont été transmis par les établissements de Montréal, ce qui peut avoir biaisé les résultats, étant donné que les habitudes et les préférences de voyage varient d'une province à l'autre.

\section{Conclusions}

Ce rapport de surveillance vise à mieux éclairer la gestion avant et après les voyages, ainsi qu'à mettre en lumière l'évolution des tendances en matière de maladies importées. Le paludisme demeure le premier diagnostic précis chez les voyageurs ayant rendu visite à des amis et à des parents, et, bien que la maladie ait été contractée en Afrique subsaharienne dans la majorité des cas, l'Inde était le premier pays source de paludisme importé dans cette cohorte. Outre le paludisme, d'autres maladies évitables contractées en voyage comme la fièvre entérique, la grippe, le virus de l'hépatite B et les morsures d'animaux étaient courantes et appuient l'idée qu'il faudrait chercher avant tout à ce que les voyageurs mettent davantage en pratique les connaissances. De plus, il faudrait évaluer les obstacles qui empêchent des groupes à risque particuliers, comme les voyageurs rendant visite à des amis et à des parents, de consulter un professionnel de la santé avant un voyage et les obstacles qui freinent le recours à des interventions préventives, comme les insectifuges et la chimioprophylaxie antipaludéenne, chez les voyageurs canadiens.

\section{Conflit d'intérêts}

II n'y a aucun conflit d'intérêts à déclarer.

\section{Références}

(1) Organisation mondiale du tourisme. Faits saillants : OMT du tourisme, Édition 2013. Accès : http://www2.unwto.org/fr

(2) Statistique Canada. Voyages effectués par les Canadiens vers les pays étrangers : 15 principaux pays visités. Statistique Canada. Tourisme et Centre de la statistique sur l'éducation, 2012. Accès : http://www.statcan.gc.ca/tables-tableaux/sum-som/l02/cst01/arts37a-fra.htm

(3) Ryan ET, Wilson ME, Kain KC. Illness after international travel. N Eng J Med 2002; 347:505-516.

(4) Ryan ET, Kain KC. Health advice and immunizations for travelers. N Eng J Med 2000; 342:17161725.

(5) Steffen R, Rickenbach M, Wilhelm U, Helminger A, Schar M. Health problems after travel to developing countries. J Infect Dis 1987; 156:84-91.

(6) Boggild AK, Geduld J, Libman M, McCarthy A, Doyle P, Ghesquiere W, Vincelette J, Kuhn S, Freedman DO, Kain KC. Travel-acquired infections and illnesses in Canadians: surveillance report from CanTravNet surveillance data, 2009-2011. Open Medicine 2014; 8(1):e20-e32. 
(7) Freedman DO, Weld LH, Kozarsky PE, Fisk T, Robins R, von Sonnenburg F, et al. Spectrum of disease and relation to place of exposure among ill returned travelers. N Eng J Med 2006; 354:119-130.

(8) Leder K, Tong S, Weld L, Kain KC, Wilder-Smith A, von Sonnenburg F, et al. Illness in travelers visiting friends and relatives: a review of the GeoSentinel Surveillance Network. Clin Infect Dis 2006; 43:1185-1193.

(9) Boggild, A.K., Ghesquiere, W., McCarthy, A., pour le Comité consultatif de la médecine tropicale et de la médecine des voyages (CCMTMV). La fièvre chez le voyageur de retour au pays : Lignes directrices pour l'évaluation initiale. Relevé des maladies transmissibles au Canada 2011; 37(ACS-3):1-15.

(10) Comité consultatif de la médecine tropicale et de la médecine des voyages (CCMTMV). Recommandations canadiennes pour la prévention et le traitement du Paludisme (Malaria) chez les voyageurs internationaux. Relevé des maladies transmissibles au Canada 2009; 35(S1):1-82. Accès : http://www.phac-aspc.gc.ca/publicat/ccdr-rmtc/09vol35/35s1/indexfra.php

(11) Salit IE, Sano M, Boggild AK, Kain KC. Travel patterns and risk behaviour of HIV-positive people travelling internationally. CMAJ 2005; 172:884-888.

(12) Correia JD, Shafer RT, Patel V, Kain KC, Tessier D, MacPherson D, et al. Blood and body fluid exposures as a health risk for international travellers. J Travel Med 2001; 8:263-266.

(13) Zuckerman JN, Stephen R. Risk of hepatitis B in travelers as compared to immunization status. J Travel Med 2000; 7:170-174.

(14) Connor BA, Jacobs RJ, Meyerhoff AS. Hepatitis B risks and immunization coverage among American travellers. J Travel Med 2006; 13:273-280.

(15) Streeton CL, Zwar N. Risk of exposure to hepatitis B and other blood-borne viruses among Australians who travel abroad. J Travel Med 2006; 13:345-350.

(16) Ward BJ, Plourde $P$, pour le Comité consultatif de la médecine tropicale et de la médecine des voyages (CCMTMV). Déclaration sur les risques d'infections transmises sexuellement chez les voyageurs. Relevé des maladies transmissibles au Canada 2006; volume 32(DCC-5):1-24.

(17) Jentes ES, Blanton JD, Johnson KJ, et al. The global availability of rabies immune globulin and rabies vaccine in clinics providing direct care to travelers. J Travel Med 2013; 20(3):148-158.

(18) Wilson ME, Weld LH, Boggild A, Keystone JS, Kain KC, von Sonnenburg F, et al. Fever in returned travellers: Results from the GeoSentinel Surveillance Network. Clin Infect Dis 2007; 44:15601568.

(19) Teo C-G. Hepatitis E. Chapter 3, Infectious Diseases Related to Travel. In: CDC Health Information for International Travel. Centers for Disease Control and Prevention, Atlanta. Accès : http://wwwnc.cdc.gov/travel/yellowbook/2014/chapter-3-infectious-diseases-relatedto-travel/hepatitis-e [consulté le 28 février 2014]. 
(20) Kamar N, Dalton HR, Abravanel F, Izopet J. Hepatitis E Virus Infection. Clin Microbiol Rev 2014; 27(1):116.

(21) Bui YG, Trépanier S, Milord F, Blackburn M, Provost S, Gagnon S. Cases of malaria, hepatitis A and typhoid fever among VFRs, Quebec (Canada). J Travel Med. 2011; 18:373-378.

(22) Labrique A, Kuniholm MH, Nelson K. 2010. The global impact of hepatitis E: new horizons for an emerging virus, p. 53-93. In: Scheld WM, Grayson ML, Hughes JM (ed), Emerging infections, $9^{\text {th }}$ ed. ASM Press, Washington, DC.

(23) Éclosion de chikungunya dans les Caraïbes (2013-2014). Agence de la santé publique du Canada. Accès : http://www.phac-aspc.gc.ca/publicat/ccdr-rmtc/14vol40/dr-rm40-02/dr-rm4002-chik-fra.php

(24) Schwartz K, Giga A, Boggild AK. Chikungunya Fever in Canada: Fever and Polyarthritis in a Returned Traveler. CMAJ 2014; [paru en ligne avant impression, 24 février 2014], DOI:10.1503/cmaj.130680

(25) Schofield S, Plourde $P$, pour le Comité consultatif de la médecine tropicale et de la médecine des voyages (CCMTMV). Déclaration relative aux mesures de protection individuelle pour prévenir les piqûres ou morsures d'arthropodes. Relevé des maladies transmissibles au Canada 2012; 38(DCC-3):1-18.

(26) Vickery JP, Tribble DR, Putnam SD, McGraw T, Sanders JW, Armstrong AW, et al. Factors associated with the use of protective measures against vector-borne diseases among troops deployed to Iraq and Afghanistan. Mil Med 2008; 173(11):1060-7.

(27) Keystone JS. Compliance with travel health recommendations. In: Dupont HI, Steffen F, editors. Textbook of travel medicine and health. London: B.C. Decker Inc.; 2001.

(28) Alon D, Shitrit P, Chowers M. Risk behaviors and spectrum of diseases among elderly travelers: A comparison of younger and older adults. J Travel Med 2010; 17(4):250-5.

(29) Pavli A, Maltezou HC. Leishmaniasis, an emerging infection in travelers. Int J Infect Dis 2010; 14(12):e1032-9.

(30) Leder K, Wilson ME, Freedman DO, Torresi J. A comparative analysis of methodological approaches used for estimating risk in travel medicine. J Travel Med 2008; 15:263-72. 